\title{
AN ESTIMATE FOR CHARACTER SUMS
}

\author{
NICHOLAS M. KATZ
}

In this note, we give estimates for a class of character sums that occur as eigenvalues of adjacency matrices of certain graphs constructed by F. R. K. Chung. Her situation is as follows. We are given a finite field $F$, an integer $n \geq 1$, an extension field $E$ of $F$ of degree $n$, and an element $x$ in $E$ that generates $E$ over $F$, i.e., an element $x$ such that $E$ is $F(x)$.

Theorem 1. Let $\chi$ be any nontrivial complex-valued multiplicative character of $E^{\times}$(extended by zero to all of $E$ ), and $x$ in $E$ any element that generates $E$ over $F$. Then

$$
\left\|\sum_{t \in F} \chi(t-x)\right\| \leq(n-1) \sqrt{\#(F)} .
$$

It turns out to be easier to consider the following more general situation. $F$ is a finite field, $n \geq 1$ is an integer, and $B$ is a finite etale $F$-algebra of dimension $n$ over $F$ (i.e., over a finite extension $K$ of $F$, there exists an isomorphism of $K$-algebras $\left.B \otimes_{F} K \simeq K \times K \times \cdots \times K\right)$. We assume given an element $x$ in $B$ that is regular in the sense that its characteristic polynomial $\operatorname{det}_{F}(T-x \mid B)$ in the regular representation of $B$ on itself has $n$ distinct eigenvalues. (In terms of the above isomorphism $B \otimes_{F} K \simeq K \times K \times \cdots \times K, x$ is regular if and only if $x \otimes 1 \simeq\left(x_{1}, \ldots, x_{n}\right)$ with all distinct components $x_{i}$. Or equivalently, $x$ is regular if and only if $B$ is equal to the $F$-subalgebra $F[x]$ generated by $x$. In the special case when $B$ is a field $F$, the element $x$ is regular if and only if $F(x)=E$.)

Theorem 2. Let $\chi$ be any nontrivial complex-valued multiplicative character of $B^{\times}$(extended by zero to all of $B$ ), and $x$ in $B$ any regular element. Then

$$
\left\|\sum_{t \in F} \chi(t-x)\right\| \leq(n-1) \sqrt{\#(F)} .
$$

Proof. The basic idea is that the theorem is an immediate consequence of Weil's estimates for one-variable character sums in the case when the $F$-algebra $B$ is completely split, and that one can reduce to this case by thinking geometrically about suitable Lang torsors.

Received by the editors February 10, 1988 .

1980 Mathematics Subject Classification (1985 Revision). Primary 11L40, 11T21. 
We begin by explaining how to view the problem geometrically. Given any finite-dimensional commutative $F$-algebra $A$, we denote by $\mathbb{A}$ the smooth affine scheme over $F$ given by " $A$ as algebraic group over $F$ "; concretely, for any $F$-algebra $R$, the group $\mathbb{A}(R)$ of $R$-valued points of $\mathbb{A}$ is $A \otimes_{F} R$. We denote by $\mathbb{A}^{\times}$the open subscheme of $\mathbb{A}$ given by " $A^{\times}$as algebraic group over $F$ "; concretely, for any $F$-algebra $R$, the group $\mathbb{A}^{\times}(R)$ of $R$-valued points of $\mathbb{A}$ is $\left(A \otimes_{F} R\right)^{\times}$. These concepts will be applied to the cases $A=B$ and $A=F$. It will be important in what follows to think of $\mathbb{A}^{\times}$as a smooth commutative group scheme over $F$, but to think of $\mathbb{A}$ only as an ambient scheme (not as a group scheme) containing $\mathbb{A}^{x}$ as an open subscheme.

Because $B^{\times}$is a smooth, geometrically connected commutative group scheme over the finite field $F$, the Lang isogeny $1-$ Frob $_{F}: B^{\times} \rightarrow B^{\times}$makes $B^{\times}$into a $B^{\times}$-torsor over itself, the "Lang torsor" $\mathbb{L}$. Let us now fix a prime number $l \neq \operatorname{char}(F)$, an algebraic closure $\bar{Q}_{l}$ of $Q_{l}$, and an isomorphism of fields $C \simeq \bar{Q}_{l}$. This isomorphism allows us to view $\chi$ as a $\bar{Q}_{l}$-valued character of $B^{\times}$, by which it makes sense to push out the Lang torsor $\mathbb{L}$ to obtain a lisse rank one $\bar{Q}_{l}$-sheaf $\mathbb{L}_{\chi}$ on $B^{\times}$which is pure of weight zero. If we denote by $j: \mathbf{B}^{\times} \rightarrow \mathbf{B}$ the inclusion, we may form the extension by zero $j ! \mathbb{L}_{\chi}$ on $\mathbf{B}$. Now consider the morphism of $F$-schemes of $f: \mathbb{F} \rightarrow \mathbf{B}$ defined by $f(t):=t-x$, and the pullback sheaf $\mathscr{F}:=f^{*}\left(j_{!} \mathbb{L}_{\chi}\right)$ on $\mathbb{F}$. The sheaf $\mathscr{F}$ is lisse of rank one and pure of weight zero on the open set $f^{-1}\left(\mathbb{B}^{\times}\right)$, and zero outside. The sheaf $\mathscr{F}$ is everywhere tamely ramified, simply because on $f^{-1}\left(\mathbb{B}^{\times}\right)$it is lisse of order dividing that of $\chi$, hence of order prime to the characteristic of $F$.

In terms of this data, the character sum in question is given by

$$
\sum_{t \in F} \chi(t-x)=\sum_{t \in f^{-1}\left(B^{\times}\right)(F)} \operatorname{Trace}\left(\text { Frob }_{t, F} \mid \mathscr{F}\right),
$$

and by the Lefschetz Trace Formula this last sum is equal to

$$
\sum_{i}(-1)^{i} \operatorname{Trace}\left(\operatorname{Frob}_{F} \mid H_{\text {comp }}^{i}\left(f^{-1}\left(\mathbb{B}^{\times}\right) \otimes_{F} \bar{F}, \mathscr{F}\right)\right) \text {. }
$$

By Weil (but expressed in the language of Deligne's paper [De]) we know that the above cohomology groups $H_{\text {comp }}^{i}$ are mixed of weight $\leq i$. For dimension reasons, $H_{\text {comp }}^{i}$ vanishes for $i>2$, and $H_{\text {comp }}^{0}$ vanishes because $\mathscr{F}$ is lisse on the incomplete curve $f^{-1}\left(\mathbb{B}^{\times}\right) \otimes_{F} \bar{F}$. It thus remains only to establish the following two facts:

(a) $H_{\text {comp }}^{2}\left(f^{-1}\left(\mathbf{B}^{\times}\right) \otimes_{F} \bar{F}, \mathscr{F}\right)=0$,

(b) $\operatorname{dim} H_{\text {comp }}^{1}\left(f^{-1}\left(\mathbb{B}^{\times}\right) \otimes_{F} \bar{F}, \mathscr{F}\right)=n-1$.

Both of these facts are geometric, i.e., they concern the situation over the algebraic closure of $F$, and hence it suffices to verify them universally in the case when the $F$-algebra $B$ is completely split. (The key point here is that our hypothesis that $\chi$ is nontrivial is stable under finite extension of scalars. 
Indeed, after extension of scalars from $F$ to any finite extension field $K$, the pullback to $\left(B^{\times}\right) \otimes_{F} K$ of $\mathbb{L}_{\chi}$ is $\mathbb{L}_{\tilde{\chi}}$, where $\tilde{\chi}$ is the character of $\left(B \otimes_{F} K\right)^{\times}$obtained from $\chi$ by composition with the norm homomorphism Norm $_{K / F}$ from $\left(B \otimes_{F} K\right)^{\times}$to $B^{\times}$. Because this norm map is surjective, the character $\tilde{\chi}$ is nontrivial provided that $\chi$ is nontrivial.)

Suppose now that $B$ is simply the $n$-fold self product of $F$ with itself. Then a nontrivial character $\chi$ of $B^{\times}$is simply an $n$-tuple $\left(\chi_{1}, \ldots, \chi_{n}\right)$ of characters of $F^{\times}$, not all of which are trivial, the regular element $x$ is just an $n$-tuple $\left(x_{1}, \ldots, x_{n}\right)$ with all distinct components $x_{i}$, the open set $f^{-1}\left(\mathbf{B}^{\times}\right)$is just the complement $\mathbb{F}-\left\{x_{1}, \ldots, x_{n}\right\}$ of the $n$ distinct points $x_{i}$ in $\mathbb{F}$, the sheaf $\mathscr{F}$ is just the tensor product of the sheaves $\left[t \mapsto t-x_{i}\right]^{*} \mathbb{L}_{\chi_{i}} \mid \mathrm{F}-\left\{x_{1}, \ldots, x_{n}\right\}$, and the sum in question is

$$
\sum_{t \in F-\left\{x_{1}, \ldots, x_{n}\right\}} \chi_{1}\left(t-x_{1}\right) \chi_{2}\left(t-x_{2}\right) \cdots \chi_{n}\left(t-x_{n}\right) .
$$

By assumption, at least one of the $\chi_{i}$ is nontrivial. For such an index $i$, the sheaf $\left[t \mapsto t-x_{i}\right]^{*} \mathbb{L}_{\chi_{i}}$ is tamely but nontrivially ramified at $x_{i}$, while all the other factors $\left[t \mapsto t-x_{j}\right]^{*} \mathbb{L}_{\chi_{j}}$ with $j \neq i$ are lisse at $x_{i}$ (by the hypothesis that all the $x_{j}$ are distinct). Therefore, the sheaf $\mathscr{F}$ is nontrivially ramified at the point $x_{i}$. Because $\mathscr{F}$ is lisse of rank one on $\mathbb{F}-\left\{x_{1}, \ldots, x_{n}\right\}$, its coinvariants under the inertia group $I_{x_{i}}$ must vanish, and a fortiori its covariants under the entire $\pi_{1}^{\text {geom }}$ of $\mathbb{F}-\left\{x_{1}, \ldots, x_{n}\right\}$ must also vanish, i.e., its $H_{\text {comp }}^{2}$ vanishes. Once we have the vanishing of all the $H_{\text {comp }}^{i}$ save for $i=1$, the asserted dimension formula $\operatorname{dim} H_{\text {comp }}^{1}=n-1$ is then equivalent to the Euler characteristic formula

$$
\sum_{i}(-1)^{i} \operatorname{dim} H_{\text {comp }}^{i}\left(\left(\mathbb{F}-\left\{x_{1}, \ldots, x_{n}\right\}\right) \otimes_{F} \bar{F}, \mathscr{F}\right)=1-n,
$$

which holds because $\mathscr{F}$ is lisse of rank one and everywhere tame on the open curve $\left(\mathbb{F}-\left\{x_{1}, \ldots, x_{n}\right\}\right) \otimes_{F} \bar{F}$, whose Euler characteristic is $1-n$. Q.E.D.

Remarks and Questions. (1) If we drop the hypothesis that the element $x$ be regular, then Theorem 2 remains valid for characters $\chi$ of $B^{\times}$whose restriction to $F^{\times}$is nontrivial. The proof proceeds along the same lines as above, reducing to the completely split case in which $\chi$ is simply an $n$-tuple $\left(\chi_{1}, \ldots, \chi_{\eta}\right)$ of characters of $F^{\times}$, with the property that their product $\prod_{i} \chi_{i}$ is nontrivial on $F^{\times}$. Now one gets the vanishing of $H_{\text {comp }}^{2}$ by observing that the sheaf $\mathscr{F}$ is nontrivially ramified at $\infty$ (as an $I_{\infty}$-representation, $\mathscr{F}$ is isomorphic to $\mathbb{L}_{\Pi_{i} \chi_{i}}$ ), and the constant " $n-1$ " actually improves to "(the number of distinct $\left.x_{i}\right)-1$." Indeed, in the case of the choice $x:=0$, the character sum in question is exactly $\sum_{t \in F \times} \chi(t)$. (Alternately, one could apply Theorem 2 directly to the (automatically finite etale) subalgebra $B_{0}:=F[x]$ of $B$ generated by $x$ over $F$, to the regular element $x$ of $B_{0}$, and to the nontrivial (because nontrivial on $F^{\times}$) character $\chi \mid\left(B_{0}\right)^{\times}$.) 
(2) What happens if we also drop the hypothesis that $B$ be etale? Suppose that we are given an arbitrary $n$-dimensional commutative $F$-algebra $A$, a multiplicative character $\chi$ of $A^{\times}$(extended by zero to all of $A$ ) whose restriction to $F^{\times}$is nontrivial, and an element $x$ in $A$. It seems plausible that the estimate

$$
\left\|\sum_{t \in F} \chi(t-x)\right\| \leq(n-1) \sqrt{\#(F)}
$$

should still hold. For example, in the case when $A$ is the algebra of dual numbers $F[x] /\left(x^{2}\right)$, the character sums in question are none other than the usual Gauss sums attached to the field $F$.

\section{REFERENCES}

[Ch] F. R. K. Chung, Diameters and eigenvalues, J. Amer. Math. Soc. 2 (1989), 187-196.

[De] P. Deligne, La conjecture de Weil II, Inst. Hautes Études Sci. Publ. Math., no. 52, 1981, pp. 313-428.

[We] A. Weil, On some exponential sums, Proc. Nat. Acad. Sci. U.S.A. 34 (1948), 204-207.

Department of Mathematics, Princeton University, Princeton, New Jersey 08544 